\title{
Individual and social determinants of oral health in South Africa in the context of COVID-19
}

SADJ September 2020, Vol. 75 No. 8 p440 - p444

\section{A Magan}

\section{INTRODUCTION}

The global pandemic due to infection with the Severe Acute Respiratory Syndrome Coronavirus 2 (SARS-CoV -2) causes the disease COVID-19 which is a mild, selflimiting disease in the majority of infected individuals. ${ }^{1}$ However, in many individuals, particularly the elderly, or those with comorbidities such as diabetes, pulmonary disease or cardiovascular conditions, infection with SARS-CoV-2 has resulted in more severe symptoms, and has proved fatal. ${ }^{2}$

Given that COVID-19 is a novel disease and that there is no vaccine or specific pharmacologic treatment for it, it is likely that its impact on an individual's general health will be protracted and is yet to unfold. Oral health is inextricably linked to general health and its neglect may have negative consequences on human and economic capital. The aim of this commentary is, therefore, to highlight the potential impact of SARS-CoV-2 on oral health in South Africa (SA).

\section{Pathogenesis and epidemiology of SARS-CoV-2}

The virus SARS-CoV-2, which most likely has a zoonotic origin, is transmissible from person-to-person either through direct contact, or via respiratory droplets produced when coughing or sneezing; ${ }^{1}$ thus, the primary portal of entry of the virus into the host is via the mucous membranes of the mouth, nose and eyes.

Entry of the SARS-CoV-2 virus into host cells is mediated by the attachment of its surface spike proteins to angiotensin-converting enzyme 2 (ACE2) receptors ${ }^{3}$ expressed by epithelial cells of the oral and nasal mucosae, lung, intestine and kidney, and the endothelium of blood vessels. ${ }^{4}$ Epidemiological data of COVID-19 cases in China, Italy, the United Kingdom (UK) and the United States of America (USA) have shown that the most common comorbidities associated with fatality were hypertension, diabetes and obesity. 2,5,6

\section{Author affiliation:}

Ansuyah Magan: BSc.(Hons), BDS, MSc.(Dent), PhD, Private Dental Practice, Honorary Research Fellow, SAMRC/Wits Developmental Pathways for Health Research Unit, Department of Paediatrics, School of Clinical Medicine, Faculty of Health Sciences, University of the Witwatersrand, Parktown, Johannesburg, South Africa. ORCID Number: 0000-0001-9183-6476

PO Box 90832, Bertsham, 2013, South Africa.

Email: ansuyah1@gmail.com
Hypertension and diabetes are commonly treated with ACE2 inhibitors; ${ }^{7}$ therefore, it has been suggested that there is greater expression of ACE2 in these patients which increases their susceptibility to a more fatal form of COVID-19. ${ }^{8}$

\section{Relationship between oral health and non-communicable diseases}

Diabetes and hypertension are non-communicable diseases (NCDs) which share modifiable risk factors with dental diseases including dental caries, periodontitis and oral cancers. These oral diseases are among the most common global diseases, ${ }^{9}$ and are caused by a high sugar content diet, tobacco use, and excessive alcohol consumption.

The World Health Organisation reported a rise in these risk factors in Africa, ${ }^{9}$ which in SA has been shown to be associated with an increase in prevalence of NCDs. ${ }^{10}$ This was highlighted in a study of 1251 African females from the Birth to Twenty cohort in Soweto, Johannesburg, in whom the prevalence of diabetes, metabolic syndrome (a cluster of cardiovascular diseases, type 2 diabetes and waist circumference), and obesity were $14 \%, 42.1 \%$ and $50.1 \%$, respectively. ${ }^{11}$

The relationship between diabetes and periodontitis is bidirectional as they share pathophysiologic pathways that include inflammation, altered host responses, and altered tissue homeostasis. ${ }^{12}$ In a study of 10 diabetic and 10 non-diabetic patients with periodontal disease, nonsurgical periodontal therapy resulted in improved glycaemic control at 3 months and 6 months follow-up. ${ }^{12}$ Although this study was limited by its small sample size, and by not having a control group that received no treatment, the findings are consistent with a recent systematic review and meta-analysis which assessed nine randomised clinical trials that included a control group with no periodontal intervention. ${ }^{13}$

The role of periodontal bacteria in the aetiology of hypertension was shown in a robust study of a total of 1056 participants. ${ }^{14}$ The subgingival biofilm consisting of A. actinomycetemcomitans, T. Forsythia, P. gingivalis, and T. denticola, described as the aetiologic burden, was positively associated with prevalent hypertension. After adjusting for age, body mass index, race, smoking, 
education, diabetes and cholesterol, the highest tertile of aetiologic burden was associated with greater systolic $(9 \mathrm{mmHg})$ and diastolic pressure $(5 \mathrm{mmHg})$ than the lowest tertile. ${ }^{14}$

In the context of COVID-19, it has not yet been established whether poor oral hygiene or periodontitis increase the risk of morbidity in patients with NCDs, and these associations need to be tested empirically. It is, however, of concern that given the high prevalence of NCDs in SA, COVID-19 may result in greater morbidity and mortality.

\section{Nutrition and food security}

South Africa has a double burden of malnutrition with a high prevalence of both obesity and undernutrition. ${ }^{15}$ Steyn and $\mathrm{Nel}^{16}$ found that fat and energy intake were greater in urban SA women than their rural peers by $13.5 \%$ and $4.6 \%$, respectively. In a more recent study of SA children aged between 1 and 8.9 years, the added sugar intakes as a proportion of total energy intake were $10.3 \%$ and $7.5 \%$ in urban and rural areas, respectively. ${ }^{17}$ While a high sugar intake is a low cost source of food energy, it is devoid of micronutrients, and is associated with the development of chronic conditions such obesity and diabetes. ${ }^{18}$ These studies reflect the nutrition transition that has accompanied urbanisation in $\mathrm{SA},{ }^{19}$ and which may have adverse consequences on oral health. In addition, it is yet to be determined whether during the current pandemic, due to lack of affordability of nutritious foods, individuals have substituted unhealthy alternative processed foods with a high content of added sugar, salt and fat, and have thereby increased their risk for caries and cardiometabolic disease.

Social and health problems tend to cluster among impoverished populations ${ }^{20}$ which is of particular significance in SA, as it has one of the highest rates of income inequity in the world. Approximately $56 \%$ of its population live in poverty and $28 \%$ in extreme poverty. ${ }^{21}$ In 2017, 6,8 million South Africans experienced hunger with 1,7 million households across the country affected. ${ }^{22}$ Thus, a high percentage of the population face food insecurity which may lead to malnutrition. Furthermore, the severe lockdown restrictions to contain the spread of SARS-CoV-2 in SA have caused a further downturn in the economy, and exacerbated the problem of food security.

Many children are dependent on school meals to sustain their nutrition; therefore, prolonged closure of schools during the pandemic with school meals no longer available, is likely to worsen the prevalence of malnourishment. Malnourished children are more susceptible to infectious diseases, and show a delay in cognitive development, which negatively impacts on educational outcomes ultimately resulting in lower income in adulthood. ${ }^{23}$

Other adverse long-term health effects of malnourishment in infancy and early childhood are stunting, ${ }^{15,24}$ and a greater risk for developing cardiometabolic diseases during the lifecourse. ${ }^{25}$ The relationship between longi- tudinal growth and oral health was shown in a recent randomised controlled trial where significantly greater height was found after a 6-month follow-up of SA children who had severe caries treated under general anaesthesia compared to their peers who had no treatment. ${ }^{26}$ Both direct and indirect (immune, endocrine and metabolic) mechanisms have been proposed to explain the relationship between untreated caries and impaired growth; ${ }^{27}$ however, these need further investigation in SA children, while considering socioeconomic contexts.

\section{Maternal factors and child oral health}

Maternal socioeconomic factors and undernutrition contribute to adverse birth outcomes such as preterm delivery or low birth weight, ${ }^{28,29}$ which have been shown to be associated with enamel hypoplasia of the deciduous and permanent dentition. ${ }^{30}$ A longitudinal study of Brazilian children aged between 12 and 36 months reported enamel defects, namely opacities and hypoplasia, in children of mothers from economically deprived communities, and suggested that this may affect their oral health related quality of life $(\mathrm{OHrQL}){ }^{30}$ The odds for enamel defects increased 3.46-fold in neonates who had had poor intrauterine nutrition and as a result were small for gestational age (SGA), and 1.89 -fold in infants who had poor postnatal nutrition. ${ }^{30}$ Maternal malnutrition also negatively impacts on the development of the stomatognathic system; ${ }^{31}$ however, there are conflicting reports in the literature on the associations between maternal malnutrition during pregnancy and the timing of tooth eruption. ${ }^{32,33}$

While these studies highlight the intergenerational impact of maternal malnutrition on child oral health, the Pelotas longitudinal study showed that poverty at birth and during the life course was correlated with the number of unsound teeth at 24 years of age. ${ }^{34}$ Early life exposures are therefore critical to an individual's health and well-being through the lifespan.

Antenatal clinics provide the ideal setting for promoting oral health; however, a recent SA study at an urban maternal and child healthcare facility found minimal integration of oral health education with general health, and that a significant number of mothers did not recognise the importance of the primary dentition. ${ }^{35}$ Interventions are essential to promote attendance of mothers at dental clinics, and to encourage preventative dental care measures once the primary teeth begin to erupt.

\section{Socioeconomic disparities in relation to oral health}

Poor oral health may cause pain and impede function. The OHrQL is a tool used to measure how an individual's oral health impacts on their general well-being and ability to function on a physical, social and psychological level. ${ }^{36}$ In a systematic review of the OHrQL in children in Africa, Malele-Kolisa et al. ${ }^{37}$ found that individual factors such as children's psyche and oral problems, except for dental caries, were associated with environmental factors such as area of residence and socioeconomic status. Thus, in SA, where there is a strong correlation between low socioeconomic status 
and ethnicity, ${ }^{38}$ sociocultural contexts should be considered when delivering oral health care.

Studies conducted in the USA ${ }^{39}$ and the $\mathrm{UK}^{6}$ have shown that black and Asian individuals with COVID-19 had double the risk of dying than their white peers. The UK cohort study included an analysis of 5683 in-hospital deaths due to COVID-19 during a 12week period, and found that pre-existing comorbidities or higher deprivation only partly accounted for death. ${ }^{6}$ It was suggested that these ethnic groups may have been over-represented as front-line workers, and therefore at a higher risk of infection, or that other social aspects such as higher household density may have been contributory factors. ${ }^{6}$ Ethnicity as a contributory factor in the spread of the SARS-CoV-2 virus in SA is deeply entrenched in the legacy of Apartheid.

The majority of South African citizens are socioeconomically disadvantaged, have poor and overcrowded living conditions, and limited access to water and sanitation. These conditions render social distancing, and maintaining stringent general and oral hygiene more challenging. Neglect of oral health may also arise due to psychological factors such as fear of entering a highrisk dental environment for treatment. However, a positive outcome of the pandemic is that individuals have become acutely aware of the importance of hygiene and the mode of transmission of infectious diseases.

In children, in particular, the greater awareness that prevails could present an opportunity to promote and reinforce positive oral health behaviour. It is important to target this age group since dental caries can be tracked; that is, the severity of caries in the primary dentition is a predictive of caries in adulthood. ${ }^{40}$ The prevalence and severity of caries in the primary dentition are also strongly related to individual and socioeconomic factors such as family income and maternal education. ${ }^{41}$

A significant number of South African households were facing financial challenges even prior to the COVID-19 pandemic due to the high unemployment rate of $30.1 \% .{ }^{42}$ The national lockdown has exacerbated the unemployment crisis with permanent closure of businesses and further job losses. A recent survey of 2688 individuals residing in SA found that, at the start of the lockdown, $5.2 \%$ of respondents had no income, and that this increased to $15,4 \%$ in the subsequent six weeks. ${ }^{43}$ Almost $75 \%$ of the respondents reduced their spending to compensate for the loss of income. For many, this has included downscaling their private medical insurance benefits. Whether this results in an increase in demand for dental care at already constrained public facilities, or that it translates to greater neglect of oral health through lack of affordability of treatment in the private sector, is yet to unfold.

\section{Oral health care workers}

The majority of South Africans have inadequate access to oral health care facilities. In SA, there are 0.13 dentists per 1000 individuals, which is fewer than most other middle-income countries. ${ }^{44}$ In addition, there are in- equities in the distribution of its oral health care workers (OHCW). ${ }^{9,44}$

While $84-90 \%$ of the SA population is reliant on public oral health services, in 2009 , only $25 \%$ of all South African dentists were employed by the public sector. ${ }^{44}$ The SA National Department of Health has demonstrated that it has the ability to garner resources and undertake intense community screening as it has for SARSCoV-2. If only such intense mobilisation could be done for the promotion of oral health screening and education in disadvantaged communities. This, together with adressing the shortage of oral hygienists and dental therapists in SA, ${ }^{45}$ could alleviate the burden of oral health care delivery in an already over-burdened environment, and should be considered by health policy advocates.

Of concern is that OHCW themselves are at a higher risk of infection by COVID-19 and may be responsible for amplification of transmission of infections. Routine screening does not identify infected patients who may be asymptomatic, or, patients may not disclose that they are infected. SARS-CoV-2 is highly contagious due to its mode of transmission, and its ability to remain viable in air droplets, and on objects and surfaces for long durations. ${ }^{46}$

There is much controversy regarding the risk posed to $\mathrm{OHCW}$ due to the aerosol generated during dental procedures even when treatment is undertaken using protocols such as a pre-procedural mouth rinse, high vacuum evacuation, rubber dam, and adequate personal protective equipment. ${ }^{47}$ It is of paramount importance that further research on airborne transmission of SARS-CoV-2 in the dental setting is undertaken so that evidence-based guidelines can be formulated for the profession.

Currently, the health risk posed to $\mathrm{OHCW}$ is compounded by the lack of availability of adequate personal protective equipment. Also, many $\mathrm{OHCW}$ in SA are older and may themselves have comorbidities and a high body mass index, and if infected, have a higher risk of suffering from a more severe form of COVID-19. Whether COVID-19 inadvertently forces older, at-risk dentists into early retirement remains to be seen. For now, practitioners have to mitigate risk by implementing additional disinfection measures, which reduces the number of patients that can be treated in a day, and by performing only those procedures which conform to the guidelines published by their professional association. Collectively, these challenges are likely to be financially crippling for many private dental practices, and compromise an already under-resourced sector.

\section{Clinical considerations of COVID-19}

There are some clinical considerations for $\mathrm{OHCW}$ when treating patients who either currently have or have recovered from COVID-19. Ibuprofen, which is a nonsteroidal anti-inflammatory drug commonly prescribed by $\mathrm{OHCW}$ for the treatment of pain, fever, and inflammation may cause an increase in ACE2. ${ }^{48}$ Since many COVID-19 positive individuals are asymptomatic, in the current environment, ibuprofen should be prescribed more ju- 
diciously. Although there are no specific drugs for the treatment of COVID-19, interferon which is known to reduce viral load, and the broad-spectrum antibiotics meropenem and moxifloxacin, are being used in some settings to treat severely ill patients. ${ }^{49}$ Intense pharmacotherapy may result in oral side effects such as xerostomia, stomatitis or candidiasis, which are known to impact on OHrQL. ${ }^{50}$ It is unclear for how long these side-effects persist after full recovery from COVID-19, and prolonged hospitalisation may exacerbate preexisting dental conditions.

Clinical and epidemiological studies have shown that patients with periodontal disease are at greater risk for developing nosocomial pneumonia, particularly during orotracheal intubation. ${ }^{51}$ The proposed mechanisms for the propensity for lung infection include aspiration of pathogenic oral bacteria into the lower respiratory tract, modification of the respiratory mucosa by salivary enzymes which facilitates bacterial colonisation, and the secretion of pro-inflammatory cytokines which promote the adhesion and colonisation of respiratory pathogens to the lung mucosa. ${ }^{51}$

Some of the risk factors for nosocomial pneumonia that need to be considered include compromised periodontal health, duration of hospital stay, antibiotic use, inadequate oral care, smoking, alcohol consumption, and advanced age. Thus, it is imperative to maintain a stringent oral hygiene regimen in the face of potential infection by SARS-CoV-2, especially for the elderly who are at greater risk for fatality due to COVID-19.

\section{CONCLUSIONS AND RECOMMENDATIONS}

The relationship between COVID-19 and oral health is indirect and multifaceted. There is a need for empirical studies to advance our understanding of the impact of COVID-19 on oral health, particularly in the South African context. The last National Oral Health survey was conducted in the early years of democracy between 1999 and 2002, and showed that approximately $45-60 \%$ of children younger than 12 years living in SA require treatment for dental decay.

In light of the nutrition transition and epidemiologic transitions $^{52}$ that have occurred in SA in the last 2 decades, an updated national survey is needed to more accurately reflect the current status of oral health in SA. In addition, prospective quantitative studies are required to determine the risks and outcomes of COVID-19 on $\mathrm{OHrQL}$ in SA, while qualitative studies may elucidate behavioural changes that may have occurred in response to the pandemic. Most importantly though, longitudinal studies that track the trajectory of oral health in relation to NCDs are critical, not only for our understanding of the complex relationship between the diseases, but also to understand their impact on human and economic capital.

Given that shared health behaviours are defined by social, environmental and political contexts, further research should be patient-centred and focus on oral health rather than oral disease. Thus, the recently developed Adult Oral Health Standard Set, ${ }^{53}$ which considers clinical, social and environmental contexts, is recommended, and would allow for clearer guidance on managing risk, and may better inform policy on delivering more appropriate oral health care and behavioural interventions in SA.

\section{References}

1. Rothan HA, Byrareddy SN. The epidemiology and pathogenesis of coronavirus disease (COVID-19) outbreak. J Autoimmun. 2020; 109: 102433.

2. Porcheddu R, Serra C, Kelvin D, Kelvin N, Rubino S. Similarity in Case Fatality Rates (CFR) of COVID-19/SARS-COV-2 in Italy and China. J Infect Dev Ctries. 2020; 14(2): 125-8.

3. Shang J, Ye G, Shi K, Wan Y, Luo C, Aihara H, et al. Structural basis of receptor recognition by SARS-CoV-2. Nature. 2020; 581(7807): 221-4.

4. Hamming I, Timens W, Bulthuis ML, Lely AT, Navis G, van Goor $\mathrm{H}$. Tissue distribution of ACE2 protein, the functional receptor for SARS coronavirus. A first step in understanding SARS pathogenesis. J Pathol. 2004; 203(2): 631-7.

5. Richardson S, Hirsch JS, Narasimhan M, Crawford JM, McGinn T, Davidson KW, et al. Presenting characteristics, comorbidities, and outcomes among 5700 patients hospitalized with COVID-19 in the New York City area. JAMA. 2020.

6. Williamson E, Walker AJ, Bhaskaran KJ, Bacon S, Bates C, Morton CE, et al. OpenSAFELY: factors associated with COVID-19-related hospital death in the linked electronic health records of 17 million adult NHS patients. medRxiv. 2020: 2020.05.06.20092999.

7. Ramos-Nino ME, Blumen SR. Benefits of ACE inhibitors in diabetes. Clin Med Therap. 2009; 1: 1041-51.

8. Fang L, Karakiulakis G, Roth M. Are patients with hypertension and diabetes mellitus at increased risk for COVID-19 infection? Lancet Respir Med. 2020; 8(4): e21.

9. WHO. Promoting oral health in Africa. 2016: 1-126.

10. Shisana O. The South African National Health and Nutrition Examination Survey, 2012: SANHANES-1: the health and nutritional status of the nation. 2014.

11. Crowther NJ, Norris SA. The current waist circumference cut point used for the diagnosis of metabolic syndrome in sub-Saharan African women is not appropriate. PLoS One. 2012;7(11):e48883.

12. Navarro-Sanchez AB, Faria-Almeida R, Bascones-Martinez A. Effect of non-surgical periodontal therapy on clinical and immunological response and glycaemic control in type 2 diabetic patients with moderate periodontitis. J Clin Periodontol. 2007; 34(10): 835-43.

13. Baeza M, Morales A, Cisterna C, Cavalla F, Jara G, Isamitt $Y$, et al. Effect of periodontal treatment in patients with periodontitis and diabetes: systematic review and meta-analysis. J Appl Oral Sci. 2020; 28: e20190248.

14. Desvarieux M, Demmer RT, Jacobs DR, Jr., Rundek T, Boden-Albala B, Sacco RL, et al. Periodontal bacteria and hypertension: the oral infections and vascular disease epidemiology study (INVEST). J Hypertens. 2010; 28(7): 1413-21.

15. Norris SA, Wrottesley S, Mohamed RS, Micklesfield LK. Africa in transition: growth trends in children and implications for nutrition. Ann Nutr Metab. 2014; 64 Suppl 2: 8-13.

16. Steyn N, Nel J. Dietary intake of adult women in South Africa and Nigeria with a focus on the use of spreads. Tygerberg: South African Medical Research Council. 2006.

17. Maunder EM, Nel JH, Steyn NP, Kruger HS, Labadarios D. Added sugar, macro- and micronutrient intakes and anthropometry of children in a developing world context. PLoS One. 2015; 10(11): e0142059.

18. Steyn NP, Temple NJ. Evidence to support a food-based dietary guideline on sugar consumption in South Africa. BMC Public Health. 2012; 12(1): 502.

19. Popkin BM, Adair LS, Ng SW. Global nutrition transition and the pandemic of obesity in developing countries. Nutr Rev. 2012; 70(1): 3-21. 
20. Mendenhall E. Syndemic suffering in Soweto: violence and inequality at the nexus of health transition in South Africa. Ann Anthropol Pract. 2014; 38(2): 300-16.

21. StatsSA. Poverty trends in South Africa. An examination of absolute poverty between 2006 and 2015 2017; Report No. 03-10-062015.

22. StatsSA. Towards measuring food security in South Africa: An examination of hunger and food inadequacy. 2019; Report: 03-00-14.

23. Stein AD, Wang M, Martorell R, Norris SA, Adair LS, Bas I, et al. Growth patterns in early childhood and final attained stature: data from five birth cohorts from low- and middleincome countries. Am J Hum Biol. 2010; 22(3): 353-9.

24. Said-Mohamed R, Micklesfield LK, Pettifor JM, Norris SA. Has the prevalence of stunting in South African children changed in 40 years? A systematic review. BMC Public Health. 2015; 15: 534

25. Victora CG, Adair L, Fall C, Hallal PC, Martorell R, Richter L, et al. Maternal and child undernutrition: consequences for adult health and human capital. Lancet. 2008; 371(9609): 340-57.

26. Yengopal $\mathrm{V}$. The effect of dental treatment on weight gain in children in South Africa [PhD]: University of the Western Cape; 2017.

27. Duijster D, Sheiham A, Hobdell MH, Itchon G, Monse B. Associations between oral health-related impacts and rate of weight gain after extraction of pulpally involved teeth in underweight preschool Filipino children. BMC Public Health. 2013; 13(1): 533.

28. Lee AC, Katz J, Blencowe H, Cousens S, Kozuki N, Vogel $\mathrm{JP}$, et al. National and regional estimates of term and preterm babies born small for gestational age in 138 low-income and middle-income countries in 2010. Lancet Glob Health. 2013; 1(1): e26-36.

29. Ngandu CB, Momberg D, Magan A, Chola L, Norris SA, Said-Mohamed R. The association between household socioeconomic status, maternal socio-demographic characteristics and adverse birth and infant growth outcomes in sub-Saharan Africa: a systematic review. J Dev Orig HIth Dis. 2019.

30. Chaves A, Rosenblatt A, Oliveira O. Enamel defects and its relation to life course events in primary dentition of Brazilian children: a longitudinal study. Community Dent Health. 2007; 24(1): 31.

31. Ferraz-Pereira KN, Toscano AE, Manhaes-de-Castro R. Effect of early undernutrition on masticatory morphophysiology: review of the literature. Arch Oral Biol. 2013; 58(11): 1735-43.

32. Elamin F, Liversidge HM. Malnutrition has no effect on the timing of human tooth formation. PLoS One. 2013; 8(8): e72274-e.

33. Mobley CC, Reifsnider E. Pregnancy, Child Nutrition, and Oral Health. In: Touger-Decker R, Sirois DA, Mobley CC, editors. Nutrition and Oral Medicine. Totowa, NJ: Humana Press; 2005; 17-30.

34. Peres MA, Peres KG, Thomson WM, Broadbent JM, Gigante DP, Horta BL. The influence of family income trajectories from birth to adulthood on adult oral health: findings from the 1982 Pelotas birth cohort. Am J Public Health. 2011; 101(4): 730-6.

35. Kolisa Y. Assessment of oral health promotion services offered as part of maternal and child health services in the Tshwane Health District, Pretoria, South Africa. Afr J Prim Health Care Fam Med. 2016;8:1-8.

36. Locker D, Allen F. What do measures of 'oral health-related quality of life'measure? Comm Dent Oral Epidemiol. 2007; 35(6): 401-11.

37. Malele-Kolisa Y, Yengopal V, Igumbor J, Nqcobo CB, Ralephenya TRD. Systematic review of factors influencing oral health-related quality of life in children in Africa. Afr J Prim Health Care Fam Med. 2019; 11(1): e1-e12.
38. Myburgh NG, Solanki GC, Smith MJ, Lalloo R. Patient satisfaction with health care providers in South Africa: the influences of race and socioeconomic status. Int J Qual Health Care. 2005; 17(6): 473-7.

39. Webb Hooper M, Napoles AM, Perez-Stable EJ. COVID-19 and racial/ethnic disparities. JAMA. 2020.

40. Sheiham A, Sabbah W. Using universal patterns of caries for planning and evaluating dental care. Caries Res. 2010; 44(2): 141-50.

41. Heilmann A, Tsakos G, Watt RG. Oral Health Over the Life Course. In: Burton-Jeangros C, Cullati S, Sacker A, Blane D, editors. A Life Course Perspective on Health Trajectories and Transitions. Cham (CH): Springer, 2015; 39-59.

42. StatsSA. South Africa unemployment rate 2000-2020 data. 2020.

43. StatsSA. Results from Wave 2 survey on the impact of the COVID-19 pandemic on employment and income in South Africa. 2020; Report-00-80-03.

44. Strachan B, Zabow T, van der Spuy Z. More doctors and dentists are needed in South Africa. S Afr Med J. 2011; 101(8): 523-8.

45. Bhayat A, Chikte U. Human resources for oral health care in South Africa: A 2018 update. Int J Environ Res Public Health. 2019; 16(10).

46. Van Doremalen N, Bushmaker T, Morris DH, Holbrook MG, Gamble A, Williamson BN, et al. Aerosol and surface stability of SARS-CoV-2 as compared with SARS-CoV-1. New Engl J Med. 2020; 382(16): 1564-7.

47. Mair AD, Korne PH. Decoding dental aerosols. Emerg Infect Dis. 2020; submitted.

48. Day M. Covid-19: ibuprofen should not be used for managing symptoms, say doctors and scientists. BMJ Publishing Group; 2020.

49. Dziedzic A, Wojtyczka R. The impact of coronavirus infectious disease 19 (COVID-19) on oral health. Oral Dis. 2020.

50. Thomson WM, Lawrence HP, Broadbent JM, Poulton R. The impact of xerostomia on oral-health-related quality of life among younger adults. Health Qual Life Outcomes. 2006; 4(1): 86.

51. Gomes-Filho IS, Passos JS, Seixas da Cruz S. Respiratory disease and the role of oral bacteria. J Oral Microbiol. 2010; 2(1): 5811.

52. Kabudula CW, Houle B, Collinson MA, Kahn K, Gómez-Olivé FX, Clark SJ, et al. Progression of the epidemiological transition in a rural South African setting: findings from population surveillance in Agincourt, 1993-2013. BMC Public Health. 2017; 17(1): 424.

53. Ni Riordain R, Glick M, Al Mashhadani SSA, Aravamudhan $\mathrm{K}$, Barrow J, Cole D, et al. Developing a standard set of patient-centred outcomes for adult oral health - an international, cross-disciplinary consensus. Int Dent J. 2020. 